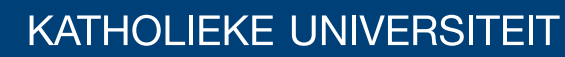 \\ LEUVEN
}

Faculty of Economics and Applied Economics

Exact algorithms for the matrix bid auction

Dries Goossens and Frits Spieksma

DEPARTMENT OF DECISION SCIENCES AND INFORMATION MANAGEMENT (KBI) 


\title{
Exact algorithms for the matrix bid auction*
}

\author{
D.R. Goossens ${ }^{(1)}$, F.C.R. Spieksma ${ }^{(1)}$ \\ (1) ORSTAT, FETEW, Katholieke Universiteit Leuven, Belgium
}

\begin{abstract}
In a combinatorial auction, multiple items are for sale simultaneously to a set of buyers. These buyers are allowed to place bids on subsets of the available items. A special kind of combinatorial auction is the so-called matrix bid auction, which was developed by Day (2004). The matrix bid auction imposes restrictions on what a bidder can bid for a subsets of the items. This paper focusses on the winner determination problem, i.e. deciding which bidders should get what items. The winner determination problem of a general combinatorial auction is NP-hard and inapproximable. We discuss the computational complexity of the winner determination problem for a special case of the matrix bid auction. We present two mathematical programming formulations for the general matrix bid auction winner determination problem. Based on one of these formulations, we develop two branch-and-price algorithms to solve the winner determination problem. Finally, we present computational results for these algorithms and compare them with results from a branch-andcut approach based on Day \& Raghavan (2006).
\end{abstract}

Keywords: Combinatorial auction, matrix bids, winner determination, computation complexity, branch-and-price

\section{Introduction}

In an auction where multiple bidders are interested in multiple items, it is often the case that the value of a set of items is higher or lower than the sum of the values of the individual items. These so-called complementarity or substitutioneffects, respectively, may be bidder-specific. A combinatorial auction is a way to make use of this synergy phenomenon. In such an auction a buyer is allowed to place bids on a subset of the items, sometimes called a bundle. The auction is concluded when the auctioneer decides to accept some of the bids and to allocate the items accordingly to the bidders.

In a combinatorial auction in its most general form, bidders can bid whatever amount they please on any subset of items. The problem of deciding which bidders should get what items in order to maximize the auctioneer's revenue is called the winner determination problem. This problem is NP-hard since (Rothkopf, Pekec \& Harstad 1998) and cannot be approximated to a ratio of $\max \left(K^{\epsilon-1}, m^{\epsilon-1 / 2}\right)$ in polynomial time for any fixed $\epsilon>0$ (unless $P=Z P P$ ),

${ }^{*}$ This research was partially supported by FWO Grant No. G.0114.03. 
where $K$ is the number of bundles on which a bid has been made, and $m$ is the number of items (Sandholm 2002). This result holds even if every item occurs in at most two bids and all prices are equal to 1 .

Numerous attempts to cope with this computational complexity can be found in literature. One approach is to impose restrictions on what a bidder can bid for these sets. A common restriction on a bidder's preferences is that they should be non-decreasing, i.e. the valuation for a set $S_{1}$ can not be higher than the valuation for a set $S_{2}$ if $S_{1}$ is a subset of $S_{2}$. Another restriction can be that the preferences should be supermodular. This means that the sum of valuations for two sets should not be higher than the sum of the valuation of the union of both sets and the valuation of the intersection of both sets. If there are only two bid functions a bidder can have, both of them non-decreasing, integer valued and supermodular, then de Vries \& Vohra (2003) point out that the winner determination problem of this auction can be solved in polynomial time. For other results based on restricting preferences, we refer to Nisan (2000) and Tennenholtz (2002).

The matrix bid auction, which was developed by Day (2004), is also a combinatorial auction in which restrictions are imposed on what a bidder can bid. In the matrix bid auction, each bidder must submit a strict ordering (or ranking) of the items in which he $\mathrm{e}^{1}$ is interested. We assume that for each bidder, the extra value an item adds to a set is determined only by the number of higher ranked items in that set, according to the ranking of that bidder. Let $G$ be the set of items, indexed by $i$ and $B$ the set of bidders, indexed by $j$. The ordering of the items is denoted by $r_{i j}$, which is item $i$ 's position in bidder $j$ 's ranking, for each $i \in G$ and $j \in B$. This ordering should be strict in the sense that for each bidder $j, r_{i_{1} j} \neq r_{i_{2} j}$ for any pair of distinct items $i_{1}$ and $i_{2}$. For instance, if $r_{i j}=2$, item $i$ is bidder $j$ 's second highest ranked item. Furthermore, each bidder $j$ specifies values $b_{i j k}$, which correspond to the value the bidder is prepared to pay for item $i$ given that it is the $k$-th highest ranked item in the set that bidder $j$ is awarded. The $b_{i j k}$ values allow to determine the value bidder $j$ attributes to any set $S \subseteq G$. Indeed, bidder $j$ 's bid on a set $S$ is denoted as $b_{j}(S)$ and can be computed as:

$$
b_{j}(S)=\sum_{i \in S} b_{i, j, k(i, j, S)}
$$

where $k(i, j, S)$ is the ranking of item $i$ amongst the items in the set $S$, according to bidder $j$ 's ranking. Notice that equation (1) assumes that no externalities are involved, i.e. a bidder's valuation depends only on the items he wins, and not for instance on the identity of the bidders to whom the other items are allocated. Furthermore, the matrix bid auction is a multi-item, single-unit combinatorial auction. This means that for each item that is auctioned, only one unit of this item is available. The winner determination problem is, given the bids $b_{j}(S)$ for each set $S$ and each bidder $j$, to determine which bidder is to receive which items, such that the total winning bid value is maximized. Notice that we assume that each bidder pays what he bids for the subsets he wins.

\footnotetext{
${ }^{1}$ he can be replaced by she (and his by her)
} 
Observe that the value for index $k$ of item $i$ in bidder $j$ 's bid can never be higher than the rank $r_{i j}$. This allows us to arrange the values $b_{i j k}$ as a lower triangular matrix for each bidder $j$, where the rows correspond to the items, ordered by decreasing rank and the columns correspond to values for $k$. Hence the name matrix bid (with order). Notice also that bidder $j$ 's ranking $r_{i j}$ does not necessarily reflect a preference order of the items. If an item is highly ranked, this merely means that its added value to a set depends on less items than the added value of a lower ranked item. Furthermore, we make no assumption regarding the $b_{i j k}$ values. Indeed, these values may be negative, e.g. to reflect the disposal cost of an unwanted item. Specifying a sufficiently large negative value can also keep the bidder from winning this item in the first place. For a more elaborate discussion of the expressiveness of matrix bids and their relation to well-known micro-economic properties, we refer to Goossens \& Spieksma (2006).

There are several reasons for investigating a combinatorial auction with matrix bids. First, bids in any practical combinatorial auction are likely to posses some structure. In literature, we find references of both theoretical structures (see e.g. Rothkopf et al. (1998), Nisan (2000), and Leyton-Brown \& Shoham (2005)) and structures in practice (see e.g. Bleischwitz \& Kliewer (2005) and Goossens, Maas, Spieksma \& van de Klundert (2007)). Capturing and understanding this structure is important, not only since it allows to develop algorithms that can be more efficient than algorithms for a general combinatorial auction, but also because it improves our understanding of various properties of an auction. The matrix bid auction, where the incremental value an item adds to a bid on a set is determined only by the number of higher ranked items in that set, imposes one such structure. Thus, the matrix bid auction offers a way of capturing structure that may be present in combinatorial auctions. Second, matrix bid auctions allow for a faster computation due to the restriction on the preferences that is assumed. Indeed, Day \& Raghavan (2006) show that the computation time for the general combinatorial auction is higher and grows much faster than for the matrix bid auction. Finally, the matrix bid auction also offers a compact way of representing preferences. Indeed, each bidder only needs to communicate an ordered list of $m$ items and $\frac{m(m+1)}{2}$ matrix bid entries, which is far less than bids for each of the $2^{m}$ possible sets of items in a general combinatorial auction. These arguments are explored in more detail in Goossens \& Spieksma (2006).

The remainder of this paper is organized as follows. In section 2 , we discuss the computational complexity of the special case of the matrix bid auction winner determination problem that arises if all bidders have the same ranking of the items. Section 3 deals with two mathematical programming formulations for the general matrix bid auction winner determination problem. Based on one of these formulations, we develop two branch-and-price algorithms to solve the winner determination problem in section 4. Finally, in section 5, we present computational results for these algorithms and compare them with results from the branch-and-cut approach by Day (2004). 


\section{Computational complexity}

The key assumption in the matrix bid auction is that for each bidder, the extra value an item adds to a set depends only on the number of higher ranked items in that set, according to the ranking of that bidder. Despite this restriction, the winner determination problem of the matrix bid auction remains $N P$-hard (Day 2004). Even if each bidder has the same ranking of the items, the matrix bid auction winner determination problem remains NP-hard. Moreover, unless $P=N P$, there exists no polynomial-time approximation scheme (PTAS) for this problem.

Theorem 1. There exists no polynomial-time approximation scheme for the winner determination problem for the matrix bid auction where all bidders have an identical ranking of the items, unless $P=N P$.

Proof. We consider the winner determination problem for the matrix bid auction where all bidders have an identical ranking. We refer to this problem as MBI. The reduction is from the 3 -dimensional matching (3DM) problem. The $3 \mathrm{DM}$ problem is described as follows: given a set $M \subseteq X \times Y \times Z$ of triples, where each of the sets $X, Y$ and $Z$ has exactly $q$ elements, find the largest matching in $M$. Kann (1991) shows that it is $N P$-hard to decide whether there exists a matching of size $q$, or whether every matching has a size of at most $(1-\delta) q$ for some fixed $\delta>0$ (see also Petrank (1994)).

Every instance of 3DM can be reduced to an MBI instance in polynomial time. Suppose that the $3 q$ elements of the sets $X, Y$, and $Z$ correspond to $3 q$ items and that each 3-element subset in $M$ corresponds to a bidder. We pick an arbitrary ordering of the items and let this be the ranking of the items for each bidder. Each bidder thus has a matrix bid with this ranking and with the following entries. The highest ranked item of the triple corresponding to the bidder gets a value of 1 in the first column, the second highest ranked item gets a value of 2 in the second column, and the third highest ranked item gets a value of 3 in the third column. All other entries get a value of zero.

If an instance of 3DM has a matching of size $q$, then the corresponding instance of MBI has a solution of value $6 q$. Indeed, a solution of 3DM consists of $q$ pairwise disjoint 3-element subsets, corresponding to $q$ bidders in MBI. Each supplier has a bid of 6 for the 3 items represented by the 3 -element subset. Accepting these bids leads to a sum of winning bids equal to $6 q$. Since every element of $X \cup Y \cup Z$ occurs exactly once in the solution of 3DM, every item will also be auctioned exactly once in the MBI solution.

If our instance of 3DM has a matching of size at most $(1-\delta) q$, at most $(1-\delta) q$ entries with value 3 in the matrix bids can be used, resulting in a MBI solution value of $(1-\delta) 6 q$. Notice that for a maximal solution value, we need to use a maximal number of entries with value 3 . The number of items remaining is $3 q-3(1-\delta) q=3 \delta q$. Each pair of these items adds at most 3 to the solution value, resulting in a maximal solution value for MBI of

$$
(1-\delta) 6 q+\frac{9 \delta q}{2}=\left(6-\frac{3}{2} \delta\right) q
$$


Consequently, a polynomial-time approximation scheme for MBI would imply that we could distinguish between instances of 3DM with a matching of size $q$ and instances where every matching has a size of at most $(1-\delta) q$, which is an NP-hard problem (Kann 1991).

Notice that it follows from theorem 1 that the winner determination problem for the matrix bid auction where bidders have an identical ranking of the items is $N P$-hard. In this theorem, the number of bidders is part of the input. In the case that the number of bidders is fixed (and we still assume identical rankings), the winner determination problem can be solved in polynomial time.

Theorem 2. The winner determination problem for a matrix bid auction with a fixed number of bidders, all having an identical ordering of the items, can be solved in polynomial time.

Proof. We will show that the winner determination problem for a matrix bid auction with a fixed number of bidders, say $n$, all having an identical ranking $r$ of the items, say $1,2, \ldots, m$, can be solved by solving a longest path problem on an acyclic graph involving $O\left(\mathrm{~m}^{n+2}\right)$ nodes and $O\left(\mathrm{~nm}^{n+2}\right)$ arcs.

This graph contains nodes indexed by $\left(i, s_{1}, s_{2}, \ldots, s_{n}, k\right)$, a source, and a sink. The index $i$ refers to item $i$ and ranges from 1 to $m$. The indices $s_{j}$, with $j \in\{1,2, \ldots, n\}$, and $k$ range from 0 to $r_{i}$, with $\sum_{j} s_{j}+k=r_{i}$. There are arcs from each node $\left(i, s_{1}, s_{2}, \ldots, s_{n}, k\right)$ to $\left(i+1, s_{1}^{\prime}, s_{2}^{\prime}, \ldots, s_{n}^{\prime}, k^{\prime}\right)$, provided that $s_{j}^{\prime} \geqslant s_{j}$ for all $j \in\{1,2, \ldots, n\}$, and that $k^{\prime} \geqslant k$. Furthermore, there is an arc from the source to each node $\left(1, s_{1}, s_{2}, \ldots, s_{n}, k\right)$, and an arc from each node $\left(m, s_{1}, s_{2}, \ldots, s_{n}, k\right)$ to the sink. The arc from node $\left(i, s_{1}, s_{2}, \ldots, s_{n}, k\right)$ to $\left(i+1, s_{1}^{\prime}, s_{2}^{\prime}, \ldots, s_{n}^{\prime}, k^{\prime}\right)$ has a cost of $b_{i+1, j, s_{j}^{\prime}}$ where $j$ is the index for which $s_{j}^{\prime}=s_{j}+1$, if $k^{\prime}=k$. If $k^{\prime}=k+1$, then this arc has a cost of zero. All arcs to the sink also have a cost of zero. The graph is depicted in Figure 1 for a setting with 2 items and 2 bidders. All arcs without indication of the corresponding cost have a cost equal to zero.

The graph described above should be interpreted as follows. Each node $\left(i, s_{1}, s_{2}\right.$, $\left.\ldots, s_{n}, k\right)$ corresponds to a state where a decision has been made on the allocation of item $i$ and all items ranked higher than $i$, with each bidder $j$ receiving $s_{j}$ items and $k$ items remaining with the auctioneer. Selecting an arc from $\left(i, s_{1}, s_{2}, \ldots, s_{n}, k\right)$ to $\left(i+1, s_{1}^{\prime}, s_{2}^{\prime}, \ldots, s_{n}^{\prime}, k^{\prime}\right)$ therefore corresponds to allocating item $i+1$ to that bidder $j$ for which $s_{j}^{\prime}=s_{j}+1$. If there is no such bidder, then item $i+1$ remains with the auctioneer (and $k^{\prime}=k+1$ ). In this way, each path from source to sink determines how the items are to be allocated, and there is a path from the source to the sink for each possible allocation.

We now sketch the equivalence between the length of a path in the graph and the value of an allocation of the items. We know that in a matrix bid, the value of adding an item $i$ to a set is determined only by the number of higher ranked items. Since the graph contains only arcs from higher ranked items to lower ranked items, the effect of adding an item $i$ to a set on the bid for this set can be determined, regardless of whatever items are added to the set further down 


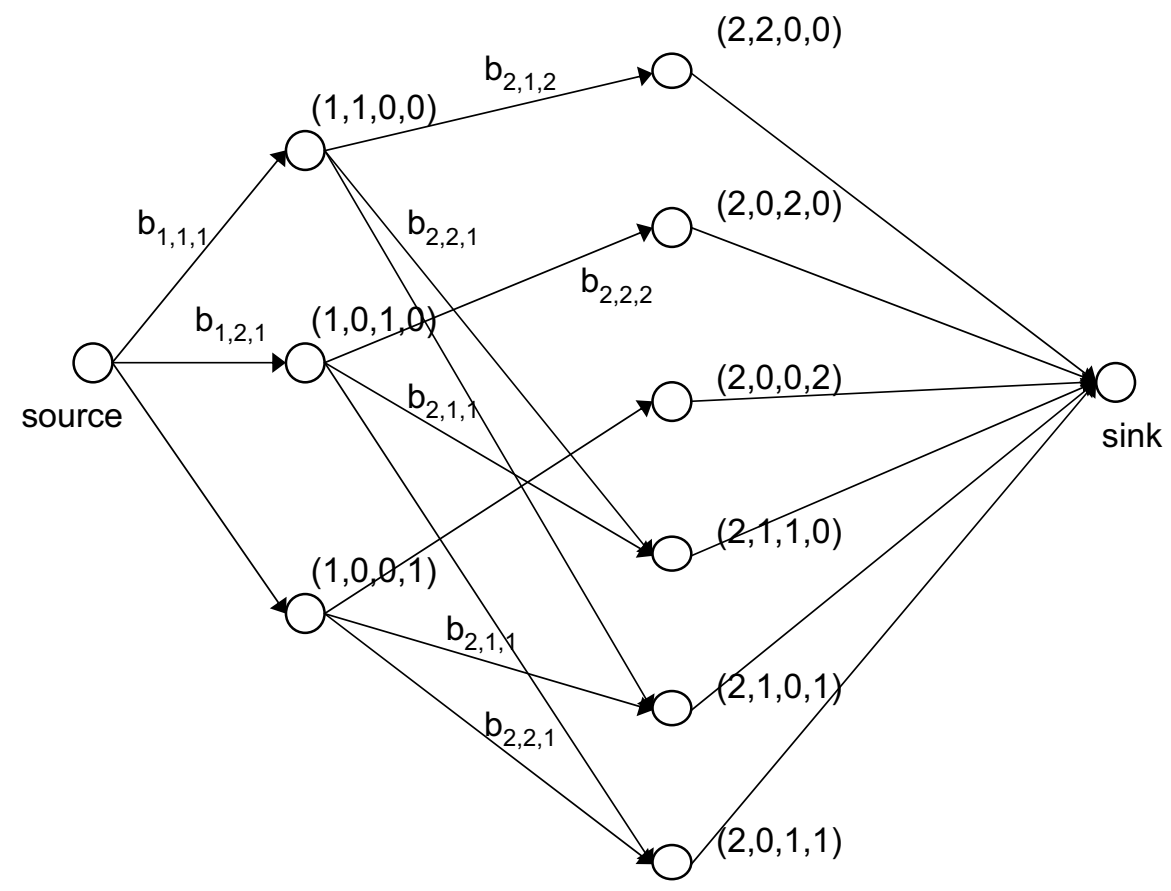

Figure 1: Illustration of the graph for 2 items and 2 bidders

the path. The cost of an arc is nothing else but the appropriate entry from the matrix bid of the bidder receiving the item. This means that the length of any path from source to sink corresponds to $\sum_{j} b_{j}\left(S_{j}\right)$, where $S_{j}$ is the set of items allocated to bidder $j$, according to that path. Therefore, the winner determination problem for a matrix bid auction with a fixed number of bidders, all having an identical ordering of the items, can be solved by solving a longest path problem. This can be done in polynomial time, since the underlying graph is acyclic.

\section{Mathematical formulations}

In this section, we present two mathematical formulations for the matrix bid auction winner determination problem. The first formulation (see also Day (2004)) is inspired by the assignment problem, the second by the set packing problem. We show that the LP-relaxations of both formulations are equally strong.

We define the binary variable $x_{i j k}$ to be 1 if bidder $j$ receives item $i$ as the $k$-th best item, and 0 otherwise. This leads to the formulation below, to which we refer as the assignment formulation. 
maximize

$$
\sum_{i \in G} \sum_{j \in B} \sum_{k=1}^{r_{i j}} b_{i j k} x_{i j k}
$$

subject to

$$
\begin{array}{ll}
\sum_{j \in B} \sum_{k=1}^{r_{i j}} x_{i j k} \leqslant 1 & \forall i \in G \\
\sum_{i \in G: r_{i j} \geq k} x_{i j k} \leqslant 1 & \forall j \in B, \forall k \in\left\{1, \ldots, r_{i j}\right\} \\
\sum_{l \in G: k \leq r_{l j} \leq r_{i j}} x_{l j k} \leqslant \sum_{l \in G: k-1 \leq r_{l j}<r_{i j}} x_{l j k-1} & \forall i \in G, \forall j \in B, \forall k \in\left\{2, \ldots, r_{i j}\right\} \\
x_{i j k} \in\{0,1\} & \forall i \in G, \forall j \in B, \forall k \in\left\{1, \ldots, r_{i j}\right\}
\end{array}
$$

Constraints (3) enforce that each item can be assigned to at most one bidder, while constraints (4) make sure that for each bidder, at most one item is the $k$-th best item in the set this bidder gets. Finally, constraints (5) impose that a bidder cannot get an item as the $k$-th best item in a set, unless a higher ranked item was assigned to this bidder as his $(k-1)$-th best item in this set. Constraints (6) are the integrality constraints.

Notice that the formulation (2)-(6) is not the minimal correct formulation for the matrix bid winner determination problem. Indeed, constraints (4) for $k \in\left\{2, \ldots, r_{i j}\right\}$ are redundant in (2)-(6), since they are already enforced by constraints (4) for $k=1$ and constraints (5). Also, replacing constraints (5) with the following (weaker) constraints still results in a correct formulation:

$$
x_{i j k} \leqslant \sum_{l \in G: k-1 \leq r_{l j}<r_{i j}} x_{l j k-1} \quad \forall i \in G, \forall j \in B, \forall k \in\left\{2, \ldots, r_{i j}\right\} .
$$

However, with this formulation, all constraints (4) remain necessary.

The set packing formulation below makes use of binary variables $y(S, j)$, which equals 1 if bidder $j$ wins set $S$, and 0 otherwise. The first set of constraints (8) enforces that each item is awarded to at most one bidder. The second set of constraints guarantees (9) that no bidder receives more than one set. The integrality constraints are (10).

maximize

$$
\sum_{j \in B} \sum_{S \subseteq G} b_{j}(S) y(S, j)
$$


subject to

$$
\begin{array}{ll}
\sum_{S \supseteq\{i\}} \sum_{j \in B} y(S, j) \leqslant 1 & \forall i \in G \\
\sum_{S \subseteq G} y(S, j) \leqslant 1 & \forall j \in B \\
y(S, j) \in\{0,1\} & \forall S \subseteq G, \forall j \in B
\end{array}
$$

Notice that this set packing formulation can also be used for the winner determination problem of a general combinatorial auction. Indeed, the matrix bid auction only differs from a general combinatorial auction in the way $b_{j}(S)$ is computed. Notice also that the assignment formulation is polynomially sized in the number of bidders and the number of items. This is not the case for the set packing formulation. In the following theorem, we prove that the LP-relaxation of the set packing formulation and the LP-relaxation of the assignment formulation are equally strong.

Theorem 3. The LP relaxation of the assignment formulation and the $L P$ relaxation of the set packing formulation are equally strong. Moreover, if the assignment formulation has an integral solution that is optimal with respect to the LP-relaxation, this is also the case for the assignment formulation, and vice versa.

Proof. In order to prove the first part of this theorem, we need to show that the LP-relaxation of the set packing formulation is at least as strong as the LPrelaxation of the assignment formulation and vice versa. In order to prove the first relation, we need to show that any solution $\hat{y}$ of the LP-relaxation of the set packing formulation can be transformed to a solution $\hat{x}$ of the LP-relaxation of the assignment formulation with the same objective function value. This is accomplished by the following procedure. For the remainder of this proof, if we mention a formulation, we mean in fact its LP-relaxation.

First, we initialize all variables $\hat{x}_{i j k}$ to 0 , for all $i \in G, j \in B$, and $k \in\left\{1, \ldots, r_{i j}\right\}$. We consider each variable $\hat{y}(S, j)$, with $S \subseteq G$ and $j \in B$ once, and set for each item $i$ in $S$

$$
\hat{x}_{i, j, k(i, j, S)} \leftarrow \hat{x}_{i, j, k(i, j, S)}+\hat{y}(S, j) .
$$

Thus, in this procedure, the value of each variable $\hat{y}(S, j)$ is added to $|S| \hat{x}_{i j k}$ variables, namely those with item $i \in S$, and $k=k(i, j, S)$. It follows that the following equality is valid:

$$
\sum_{k=1}^{r_{i j}} \hat{x}_{i j k}=\sum_{k=1}^{r_{i j}} \sum_{S: i \in S \wedge k=k(i, j, S)} \hat{y}(S, j)=\sum_{S \supseteq\{i\}} \hat{y}(S, j) \quad \forall i \in G, j \in B .
$$

Using this equality, we verify that (3) holds for $\hat{x}$ : 


$$
\sum_{j \in B} \sum_{k=1}^{r_{i j}} \hat{x}_{i j k}=\sum_{j \in B} \sum_{S \supseteq\{i\}} \hat{y}(S, j) \leqslant 1 .
$$

Notice that the last inequality follows from the feasibility of $\hat{y}$ (see (8)). We also establish for $j \in B$, and $k \in\left\{1, \ldots, r_{i j}\right\}$ :

$$
\begin{aligned}
\sum_{i \in G: r_{i j} \geqslant k} \hat{x}_{i j k} & =\sum_{i \in G} \sum_{S: i \in S \wedge k(i, j, S)=k} \hat{y}(S, j) \\
& =\sum_{S:|S| \geqslant k} \hat{y}(S, j) \\
& \leqslant \sum_{S \subseteq G} \hat{y}(S, j) \leqslant 1
\end{aligned}
$$

which shows that $\hat{x}$ satisfies (4). Finally, we have that for each $i \in G, j \in B$, and $k=1, \ldots, r_{i j}$ :

$$
\sum_{l \in G: k \leq r_{l j} \leq r_{i j}} \hat{x}_{l j k}=\sum_{l \in G: r_{l j} \leqslant r_{i j}} \sum_{S: l \in S \wedge k(l, j, S)=k} \hat{y}(S, j) .
$$

Thus we can write for each $i \in G, j \in B$, and $k=2, \ldots, r_{i j}$ :

$$
\begin{aligned}
& \sum_{l \in G: k \leq r_{l j} \leq r_{i j}} \hat{x}_{l j k}-\sum_{l \in G: k-1 \leq r_{l j}<r_{i j}} \hat{x}_{l j k-1}= \\
& \sum_{l \in G: r_{l j} \leqslant r_{i j}} \sum_{S: l \in S \wedge k(l, j, S)=k} \hat{y}(S, j)-\sum_{l \in G: r_{l j} \leqslant r_{i j}} \sum_{S: l \in S \wedge k(l, j, S)=k-1} \hat{y}(S, j) .
\end{aligned}
$$

Consider some $\hat{y}(S, j)$ occurring in the first term. The corresponding set $S$ has at the $k$-th position $(k \geqslant 2)$ some item $l, r_{l j} \leqslant r_{i j}$. It follows that there must be some other item, say $l^{\prime}$ with $r_{l^{\prime} j} \leqslant r_{l j}$ at position $k-1$. Hence this $\hat{y}(S, j)$ also occurs in the second term. It follows that the expression (16) cannot have a positive value, and hence (5) is satisfied. Notice also that the transformation procedure (11) does not affect the objective function value. Moreover, it transforms any integral solution $\hat{y}$ to an integral solution $\hat{x}$.

Hence, we have shown that the set packing formulation is at least as strong as the assignment formulation and if the set packing formulation has an integral solution that is optimal with respect to the LP-relaxation, this is also the case for the assignment formulation. In the remainder of this proof, we show that the assignment formulation is at least strong as the set packing formulation. In order to prove this second relation, we show that any solution $\hat{x}$ of the LP-relaxation of the assignment formulation can be transformed to a solution $\hat{y}$ of the LP-relaxation of the set packing formulation with the same objective function value. This is accomplished by the following procedure, $\operatorname{CONVERT}(\hat{x})$. 


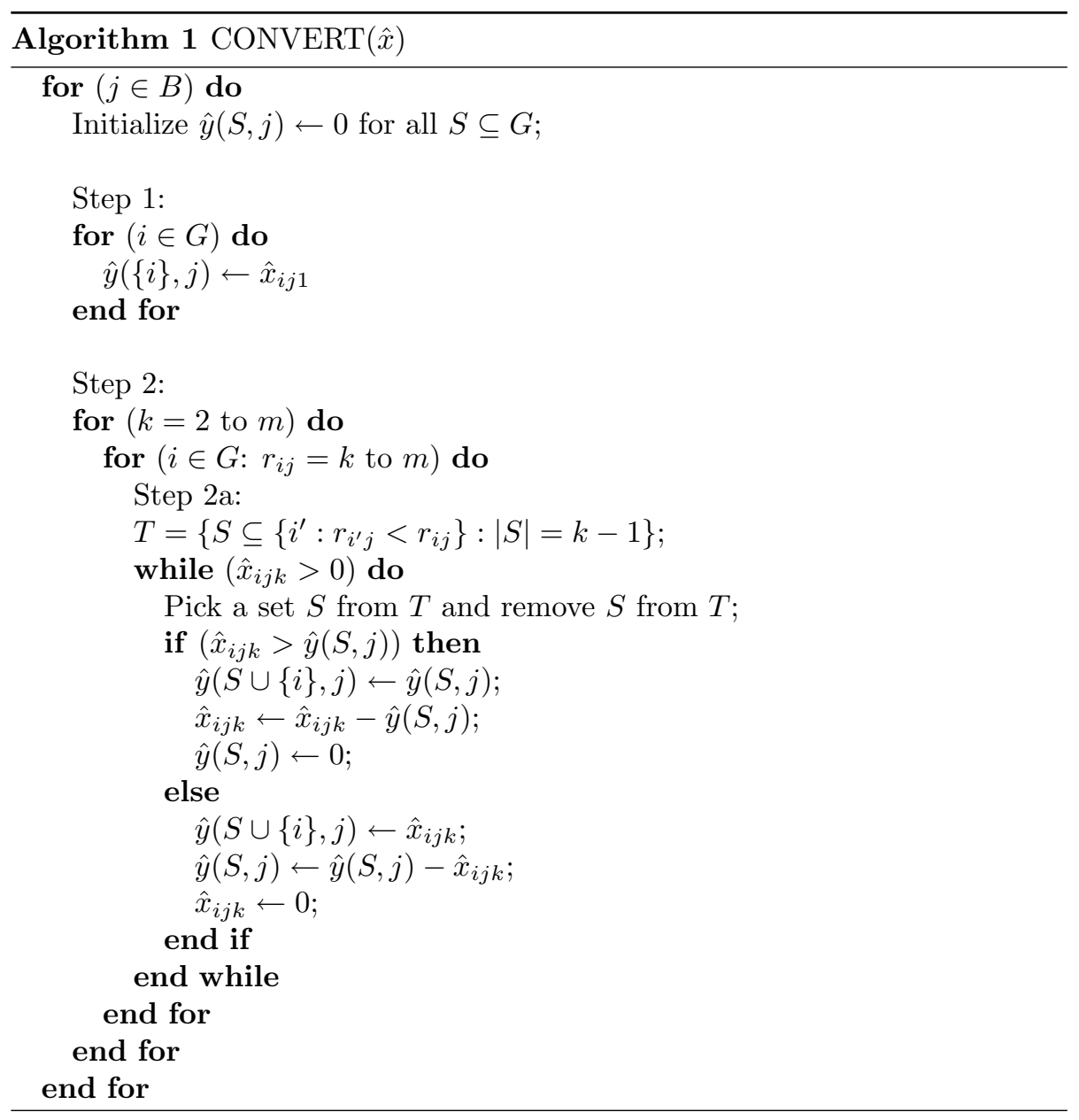


The CONVERT procedure translates any solution for the assignment formulation to a solution for set packing formulation. First, we argue that the CONVERT algorithm terminates.

The crucial step in the CONVERT algorithm is step 2a, which has to be performed for each bidder $j$, for each $k$ from 2 to $m$, and for each $i \in G$ with $r_{i j} \geqslant k$. Let us consider now a bidder $j$, item $i$, and rank $k$, for which step $2 \mathrm{a}$ is to be performed, and let $\widetilde{y}(S, j)$ be the solution as it is constructed by the CONVERT algorithm so far. In order to guarantee that the while loop in step 2a terminates, we need:

$$
\hat{x}_{i j k} \leqslant \sum_{S: S \subseteq\left\{i^{\prime}: r_{i^{\prime} j}<r_{i j}\right\} \wedge|S|=k-1} \widetilde{y}(S, j) .
$$

Notice that in CONVERT, so far, each variable $\widetilde{y}(S, j)$, with $|S|=k-1$ and $l$ being the lowest ranked item in $S$, has been increased at most once, namely with (a fraction of) $\hat{x}_{l, j, k-1}$. Furthermore, the total value of $\hat{x}_{l, j, k-1}$ has been added exclusively over variables $\widetilde{y}(S, j)$ with $|S|=k-1$ and $l$ the lowest ranked item in $S$. Therefore, we have that the total fraction that has been added to variables $\widetilde{y}(S, j)$ with $S$ containing $k-1$ items ranked higher than $i$ equals:

$$
\sum_{i^{\prime}: k \leqslant r_{i^{\prime} j}<r_{i j}} \hat{x}_{i^{\prime}, j, k-1}
$$

Notice that the value of each variable $\widetilde{y}(S, j)$ may also have been decreased in CONVERT. Indeed, variables $\widetilde{y}(S, j)$ with $S$ containing $k-1$ items and the one with the lowest rank being $l$, can be decreased only with (a fraction of) variables $\hat{x}_{i^{\prime}, j, k}$ with $i^{\prime}$ ranked higher than $l$, and lower than $i$ (since step 2a has not yet been performed for rank $k$ and item $i$ or items ranked lower than $i$ ). Furthermore, the total value of $\hat{x}_{l, j, k}$ has been subtracted only from variables $\widetilde{y}(S, j)$, with $S$ containing $k-1$ items, all ranked higher than $l$. Therefore, we have that the total fraction that has been subtracted from variables $\widetilde{y}(S, j)$ with $S$ containing $k-1$ items ranked higher than $i$ equals:

$$
\sum_{i^{\prime}: k \leqslant r_{i^{\prime} j}<r_{i j}} \hat{x}_{i^{\prime}, j, k}
$$

Thus,

$$
\sum_{S: S \subseteq\left\{i^{\prime}: r_{i^{\prime} j}<r_{i j}\right\} \wedge|S|=k-1} \widetilde{y}(S, j)=\sum_{i^{\prime}: k \leqslant r_{i^{\prime} j}<r_{i j}} \hat{x}_{i^{\prime}, j, k-1}-\sum_{i^{\prime}: k \leqslant r_{i^{\prime} j}<r_{i j}} \hat{x}_{i^{\prime}, j, k} .
$$

Further, it follows from (5) that

$$
\hat{x}_{i j k} \leqslant \sum_{i^{\prime}: k \leqslant r_{i^{\prime} j}<r_{i j}} \hat{x}_{i^{\prime}, j, k-1}-\sum_{i^{\prime}: k \leqslant r_{i^{\prime} j}<r_{i j}} \hat{x}_{i^{\prime}, j, k}
$$

for each bidder $j$, for each $k$ from 2 to $m$, and for each $i \in G$ with $r_{i j} \geqslant k$. From (20) and (21) we conclude that (17) is true and hence the CONVERT algorithm terminates. 
We now argue that solution $\hat{y}$ is indeed feasible with respect to constraints (8), (9), and the relaxation of (10).

For each bidder $j$ and each item $i$, it is clear that after step $1, \sum_{S \supseteq\{i\}} \hat{y}(S, j)=$ $\hat{x}_{i j 1}$. In step 2 , each value $\hat{x}_{i j k}$ is spread over one or more variables $\hat{y}(S, j)$ with $S$ containing item $i$. Also, for each variable $\hat{y}(S, j)$ that is increased, a variable $\hat{y}(S \backslash\{i\}, j)$ is decreased with the same value. Therefore, after step 2, $\sum_{S \supseteq\{i\}} \hat{y}(S, j) \leqslant \sum_{k=1}^{r_{i j}} \hat{x}_{i j k}$. Summing over the bidders gives

$$
\sum_{j \in B} \sum_{S \supseteq\{i\}} \hat{y}(S, j) \leqslant \sum_{j \in B} \sum_{k=1}^{r_{i j}} \hat{x}_{i j k} .
$$

Given (3), this implies that constraints (8) are satisfied.

For each bidder $j$, it is clear that after step 1 of CONVERT, $\sum_{S \subseteq G} \hat{y}(S, j)=$ $\sum_{i \in G} \hat{x}_{i j 1}$. In step 2 , for every variable $\hat{y}(S, j)$ whose value is increased, there is some other variable $\hat{y}\left(S^{\prime}, j\right)$ whose value is reduced by the same amount. Given (4), this implies that constraints (9) are satisfied.

Each variable $\hat{y}(S, j)$ is increased by at most one variable $\hat{x}_{i j k}$. Therefore, it follows from the relaxation of constraints $(6)$ that $\hat{y}(S, j) \leqslant 1$ for all $S \subseteq G$ and each bidder $j$. By construction of the algorithm, no variable $\hat{y}(S, j)$ will have a value less than zero. Thus, $\hat{y}$ satisfies the relaxation of constraints (10).

Further, the objective function value of both solutions $\hat{x}$ and $\hat{y}$ is the same. Consider any bidder $j$. After step 1, the objective function of solution $\hat{y}$ has a value equal to $\sum_{i \in G} b_{i j 1} \hat{x}_{i j 1}$, since $b(\{i\}, j)=b_{i j 1}$. Every time step $2 \mathrm{a}$ is performed, the objective function value is increased by $(b(S \cup\{i\}, j)-b(S, j)) \hat{x}_{i j k}$. Since set $S$ contains only items ranked higher than item $i$, we have $b(S \cup\{i\}, j)-b(S, j)=$ $b_{i j k}$, where $k$ is the number of items in $S$ plus one. Therefore, after step 2 the objective function equals $\sum_{i \in G} \sum_{k=1}^{r_{i j}} b_{i j 1} \hat{x}_{i j 1}$. Summing over all bidders $j$ shows that the CONVERT $(\hat{x})$ procedure produces a solution $\hat{y}$ with the same objective function value as $\hat{x}$.

Finally, it is easy to see that if the CONVERT procedure is confronted with an integral solution $\hat{x}$, it will produce an integral solution $\hat{y}$. Thus, we can conclude that the assignment formulation and the set packing formulation are equally strong, and that if one formulation has an integral optimal solution, this is also the case for the other formulation.

\section{Branch-and-price algorithms for solving the matrix bid auction}

Theorem 3 shows that the set packing formulation (7)-(10) is equally strong as the assignment formulation (2)-(6). Here we outline an algorithm based on the set packing formulation. Solving the LP-relaxation of the set packing formulation is however not trivial, given the huge amount of variables $\left(n 2^{m}\right)$. 
Considering that only a small percentage of these variables are nonzero in an optimal solution, column generation suggests itself as an efficient solution technique. Column generation was proposed by Dantzig \& Wolfe (1960) and starts by solving the LP-relaxation considering only a restricted subset of the variables. This problem is also called the restricted master problem. Notice that this problem can be restricted to $m+n$ variables, whereas the assignment formulation requires $n m(m+1) / 2$ variables, which may still be large. The next step is to verify whether any of the variables that were not considered could improve the current solution. In other words, we search for a variable with a non-negative reduced cost. This problem is called the pricing problem. If we find such a variable, we add it to the restricted master problem and solve it again. This re-optimizing and pricing is to be repeated until the pricing problem fails to produce new variables, indicating that the LP-relaxation has been solved to optimality.

Notice that the column generation procedure does not guarantee to find an integral solution. In case of a fractional solution, a branching decision needs to be made, partitioning the solution space in order to create a number of smaller subproblems. With branch-and-price, this results in a search tree where column generation has to be applied in every node. In this way, branch-and-price can be seen as a generalization of the column generation technique for integer programming. Combining the column generation approach with a branching scheme may not be straightforward. The key to an efficient branch-and-price algorithm is an easy-to-solve pricing problem. The branching rule should therefore not destroy the structure of the pricing problem or increase its complexity when moving deeper down the search tree.

Branch-and-price has proven to be successful for solving huge integer programs arising from a number of combinatorial problems (for an overview, we refer to Barnhart, Johnson, Nemhauser, Savelsbergh \& Vance (1998)). We refer to Vanderbeck \& Wolsey (1996) for a more elaborate description of the branchand-price technique. In section 4.1, we show how the LP-relaxation of the set packing formulation for the matrix bid auction winner determination problem can be solved efficiently using column generation. Next, the column generation approach is used as a building block for two branch-and-price algorithms to solve the matrix bid auction. The algorithm in section 4.2 makes use of a branching rule based on assigning items to bidders, whereas in section 4.3 , branching is done by deciding on the succession of items in a winning set. Finally, in section 4.4 , we comment on some issues that turn out to be important while implementing both branch-and-price algorithms.

\subsection{Column generation for the matrix bid auction}

In this section, we show how the LP-relaxation of the set packing formulation of the matrix bid winner determination problem can be solved using column generation. We also prove that the pricing problem can be solved in polynomial time, since it can be solved by solving a shortest path problem. 
If we define $u_{i}$ for each item $i \in G$ as the dual price associated with the corresponding constraint of (8), and $v_{j}$ for each bidder $j \in B$ as the dual price associated with the corresponding constraint of (9), we can write the dual of the set packing formulation (7)-(10) as follows:

minimize

$$
\sum_{i \in G} u_{i}+\sum_{j \in B} v_{j}
$$

subject to

$$
\begin{array}{ll}
\sum_{i \in S} u_{i}+v_{j} \geqslant b_{j}(S) & \forall S \subseteq G, \forall j \in B \\
u_{i} \geqslant 0, v_{j} \geqslant 0 & \forall i \in G, \forall j \in B
\end{array}
$$

We start by finding an optimal solution for the restricted master problem, i.e. the LP-relaxation of (7)-(10) considering only a limited number of variables $y(S, j)$. This solution is also an optimal solution for the (unrestricted) LPrelaxation of (7)-(10) if its corresponding dual variables form a feasible solution for (22)-(24), which has a constraint for every variable $y(S, j)$. Consequently, we need to add a new column or variable to the restricted master problem if a constraint of (23) is violated. The pricing problem thus boils down to determining the existence of a set $S$ of items and a bidder $j$ such that

$$
\sum_{i \in S} u_{i}<b_{j}(S)-v_{j} .
$$

Theorem 4. The pricing problem, i.e. finding a set $S$ of items and a bidder $j$ such that a constraint of (23) is violated, can be solved by solving a shortest path problem.

Proof. We construct a graph with a source and a sink, and a subgraph for each bidder $j$. Such a subgraph contains $r_{i j}$ nodes for each item $i$, called item nodes. We will refer to an item node as $(i, j, k)$, where $i$ stands for the item and $k$ ranges from 1 to $r_{i j}$. There are arcs from each node $(i, j, k)$ to each node $\left(i^{\prime}, j, k+1\right)$ where item $i^{\prime}$ is ranked lower than item $i$ (i.e., $r_{i^{\prime} j}>r_{i j}$ ). These arcs have a cost equal to $u_{i^{\prime}}-b_{i^{\prime}, j, k+1}$. Notice that there are no arcs between nodes corresponding to different subgraphs. Furthermore, for each subgraph, there are arcs from the source node to node $(i, j, 1)$ for each item $i$ with a cost equal to $u_{i}-b_{i j 1}$ and there are arcs from each item node $(i, j, k)$ to the sink with cost $v_{j}$. A schematic representation of this graph is given in Figure 2 for a setting with a single bidder $j$ and three items.

From the structure of this graph, it follows that all nodes of a path from the source to the sink correspond to the same bidder and each path contains at most one node per item. Moreover, exactly one arc with cost $v_{j}$ is included in the path. Therefore, the length of a path containing nodes $(i, j, k)$ of the items $i \in S$ of bidder $j$ in this graph equals 


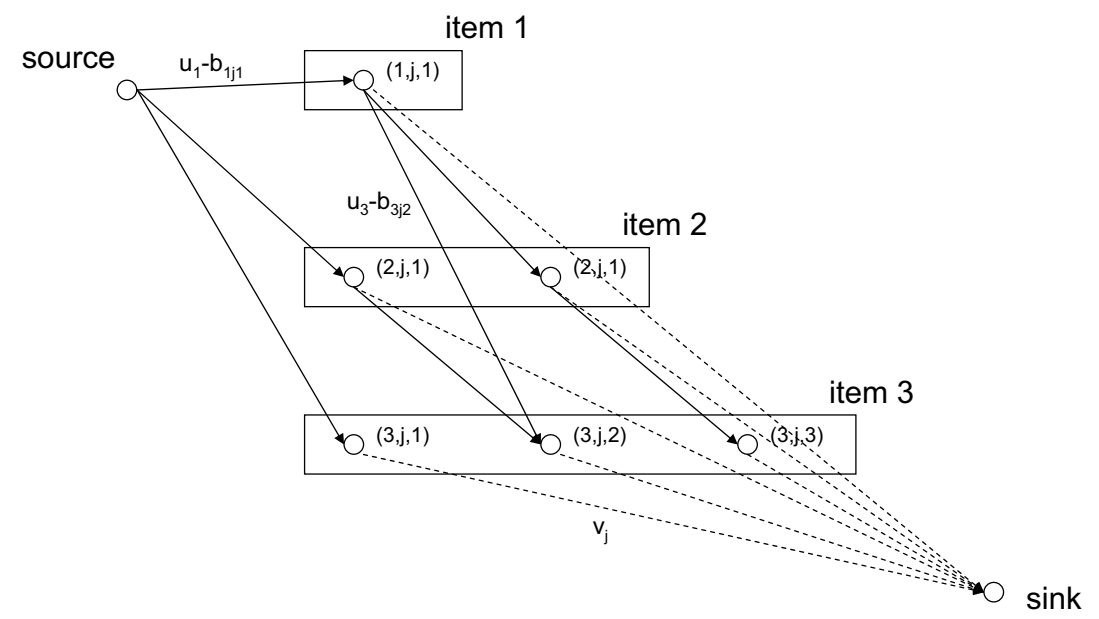

Figure 2: The pricing problem as a shortest path problem

$$
\sum_{i \in S}\left(u_{i}-b_{i j k}\right)+v_{j}
$$

Furthermore, the graph ensures that an item $i$ is in the path using its $k$-th node only if a higher ranked item is in the path through its $(k-1)$-th node. We can therefore state that $\sum_{i \in S} b_{i j k}=b_{j}(S)$ and it follows that the existence of a path with negative length corresponds to a violated constraint in the dual. Consequently, we need to solve a shortest path problem on an acyclic graph in order to solve the pricing problem.

Thus, if the shortest path has a negative length, we can add a column for the corresponding bidder $j$ containing the items in set $S$ determined by the item nodes traversed in the path. Naturally, bidder $j$ 's bid for this set $S$ is $b_{j}(S)$. Notice that since the pricing problem is solvable in polynomial time, the LPrelaxation of the set packing formulation for the matrix bid auction can also be solved in polynomial time.

Corollary 1. The LP-relaxation of the set packing formulation (7)-(10) for the matrix bid auction winner determination problem can be solved in polynomial time.

\subsection{Branching on an item-bidder pair}

The solution of the LP-relaxation of the matrix bid winner determination problem found by column generation may not be integral. If this is the case, we 
need to partition the solution space to eliminate this fractional solution. In this approach, we partition the solution space by the branching decision whether or not to assign an item to a bidder. We first prove that in a fractional solution, there always exists an item that has been fractionally assigned to one or more bidders.

Lemma 1. For any fractional solution to the relaxation of (7)-(10),

$$
\exists i \in G, j \in B: 0<\sum_{S: S \supseteq\{i\}} y(S, j)<1
$$

Proof. We will prove this theorem by showing that a solution must be integral if it does not satisfy (27). Consider a solution for which property (27) is not valid. This means that each item has been assigned fully or not at all to each bidder. In this case, no items are split over multiple bidders. An item $p$ for which $\sum_{S: S \supseteq\{p\}} y(S, j)=1$ could, however, still be split over multiple sets of the same bidder $j$. It is easy to see that if bidder $j$ is awarded a set $S$ containing next to $p$ any other item $q$, that this item then should occur in each set containing $p$ in order to have the sum of the fractions of sets containing $p$ equal 1. In other words, the sets of bidder $j$ are identical, and we have, in fact, an integral solution.

The branch-and-price algorithm can, however, only be valid if in every node of the search tree, all generated columns satisfy the previously made branching decisions. Prohibiting that an item is awarded to a certain bidder in the pricing problem can be done by simply removing the vertices corresponding to that item for that bidder from the graph. Enforcing that an item is awarded to a certain bidder in the pricing problem is less obvious. For that bidder, the arcs from the source to any lower ranked item need to be removed. Also the arcs from any higher ranked item to any item ranked lower than that item need to be deleted. Finally, the arcs from the higher ranked items to the sink must be removed as well. Clearly, all nodes that can no longer be reached as a consequence of these removals can now also be deleted, as are the arcs leaving those nodes, and so on. For all other bidders, we need to remove the vertices of that item from the graph. Figure 3 shows the pricing problem where item 2 is forced to be awarded to the bidder whose item nodes are depicted. In this graph, we made sure that every path from the source to the sink of that bidder must include a node corresponding to item 2 .

Notice that this branching rule does not destroy the structure of the pricing problem: in all branches, the pricing problem remains a shortest path problem. It is easy to see that this shortest path problem can be adjusted to produce columns that comply with a series of branching decisions. Moreover, when moving deeper down the tree, more and more arcs and nodes will be removed. Thus, we have described a valid branching rule where the pricing problem remains solvable as a shortest path problem throughout the search tree.

\subsection{Branching on a pair of successive items}

Ryan \& Foster (1981) suggest a branching rule for the set partitioning problems 


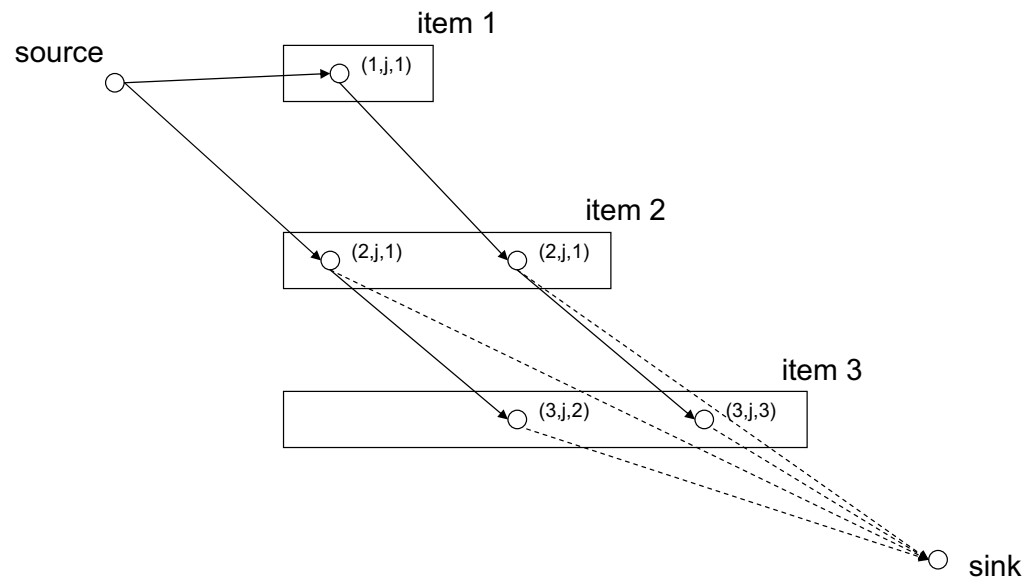

Figure 3: The pricing problem where the bidder must get item 2

where two constraints are covered together or not at all by the variables in one branch, whereas in the other branch, each variable can cover at most one of these constraints. This rule can easily be generalized to set packing problems and can be translated to a combinatorial auction context as two items needing to go to the same bidder in one branch and to different bidders in the other branch. However, forcing two arbitrary items to go to the same bidder, but also forbidding that these items go to the same bidder, is not straightforward to achieve in the shortest path problem described in section 4.1. Therefore, we modify this branching rule, such that it takes into account the ranking of the items specified in the bidder's matrix bid. A similar modification has been applied for example in a pallet loading application (Moonen 2005).

We partition the solution space by branching on a pair of items $p$ and $q$. In one branch, we enforce that if item $p$ is present in a bidder's set, then item $q$ must be directly successive to $p$ in this set, when the set is sorted according to this bidder's ranking of the items. In the other branch, no bidder can have items $p$ and $q$ as direct successors in a set, according to his ranking. We first prove that there always exists a pair of items such that the sets in which these items occur as direct successors according to the corresponding bidder's ranking, have been fractionally assigned to one or more bidders. We introduce the notation $p \rightarrow_{j} q$ to denote that item $p$ is directly succeeded by item $q$ in a set, according to the ranking of bidder $j$.

Lemma 2. For any optimal, extreme fractional solution to the relaxation of (7)-(10),

$$
\exists p, q \in G: 0<\sum_{j \in B} \sum_{S: S \supseteq\{p, q\} \wedge p \rightarrow{ }_{j} q} y(S, j)<1
$$


Proof. Assume that we have an optimal, extreme fractional solution for which (28) is not satisfied. This means that for each pair of items, each bid on a set in which these items are direct successors according to ranking of the bidder that made the bid, has been assigned to that bidder for a total fraction of 0 or 1 . Thus, for any items $p$ and $q$ for which $\sum_{j \in B} \sum_{S: S \supseteq\{p, q\} \wedge p \rightarrow_{j} q} y(S, j)=1$, we can conclude that if item $p$ is present in a set, that then also item $q$ is present in this set. Therefore, each pair of sets to which a positive fraction has been assigned is disjoint or identical. Since there is a single variable $y(S, j)$ representing identical sets of the same bidder $j$, we conclude that identical sets must be split over multiple bidders. This leaves us with the problem of assigning a number of disjoint sets among one or more bidders, where each assignment of a set to a bidder has its profit, namely the bid of this bidder for this set. This problem is a maximum weighted assignment problem on a bipartite graph, where each node on one side of the partition represents a set, and each node on the other side of the partition represents a bidder. It follows that each optimal, extreme solution is integral. Consequently, for any optimal, extreme fractional solution to the relaxation of (7)-(10), property (28) is true.

The above theorem shows that it is always possible to find a pair of items $p$ and $q$ on which to branch. However, we still need to enforce that the pricing problem will generate columns that satisfy the constraint imposed by the branching decision. In the branch where we impose $p \rightarrow_{j} q$, for each bidder $j$, we need to remove all arcs from nodes corresponding to $p$ to any node not corresponding to $q$. Notice that for a bidder that ranks $q$ higher than $p$, this comes down to removing all nodes related to $p$ from the graph. This leaves us with a graph where if one arrives in a node related to $p$, the only option is to take an arc to a node related to $q$. In the branch where $p$ should not be directly succeeded by $q$, it suffices, for each bidder, to remove the arcs going from a $p$-node to a $q$-node, if they exist.

Notice this branching rule does not destroy the structure of the pricing problem either, even when we consider a sequence of branching decisions. Indeed, it is not hard to verify that when going deeper into the search tree, the pricing problem can still be solved as a shortest path problem on an increasingly smaller graph.

\subsection{Implementation issues}

Both branch-and-price algorithms were implemented using Visual $\mathrm{C}++6.0$. The set packing problems were solved using Ilog Cplex 8.1. The LEDA libraries (version 5.0.1) allowed us to solve the shortest path problems in linear time. In the remainder of this section, some of the most important implementation issues are discussed.

\subsubsection{Solving the root node}

A first issue that needs to be solved is determining which columns will be used in the very first restricted master problem. Using many columns obviously increases the computation time needed to solve the restricted master problem. On 
the other hand, this may result in a solution that is closer to the optimal solution, such that less iterations for solving the pricing problem and re-optimizing are needed. In our case, after experimenting with a number of settings, it turned out that including a rather large number of variables to start the column generation process pays off. We constructed a set for every strictly positive entry in the matrix bid by taking the item corresponding to this entry and completing the set with the $k$ highest ranked items, where $k$ is the entry's column in the matrix bid.

After the restricted master problem has been solved and the corresponding dual solution has been obtained, new columns with a non-negative reduced cost need to be added. The question remains how many such columns we should add. Again, adding too many new variables increases the computation time for solving the resulting restricted master problem, whereas adding too few variables can result in a large number of iterations for solving the pricing problem and re-optimizing. The strategy that proved to be the most efficient consists of adding for each bidder those variables whose reduced cost is at most $2 \%$ less than the most positive reduced cost for a variable from that bidder. Furthermore, the number of such variables that is added for each bidder cannot exceed the number of items. Notice that finding these variables demands very little extra computation time, since the LEDA libraries provide the distance from the source to each node in the graph, after having solved the shortest path problem.

Finally, when re-optimizing the restricted master problem, we start from the optimal base of the previous iteration. In order not to drag along too many columns for the remainder of the search tree, those columns that were added at some iteration, but never made part of any base solution are removed from the model. We keep the other columns, assuming that they will be useful again later.

\subsubsection{A selection rule when branching on an item-bidder pair}

The major issue in implementing this branching rule is to choose the item on which to branch and the bidder(s) to assign it to. We chose to branch on the item that is fractionally assigned to the highest number of bidders. For each of these bidders, a branch is constructed in which the bidder is assigned the item. A final branch is added where none of these bidders is allowed to receive the item. We opted for a depth-first strategy, where the branch where the item is assigned to the bidder with the highest fraction is explored first. Thus, the branch where bidders are disallowed to receive an item always comes last.

\subsubsection{A selection rule when branching on a pair of successive items}

With this branching rule, each node that needs further partitioning of the solution space leads to two branches. In the first branch, we enforce that for each bidder, if item $p$ is present in a bid, $q$ should be the next item in that bid, according to the ranking of that bidder. The second branch considers only bids for which $p$ and $q$ are no direct successors according to the bidder's ranking. We again chose a depth-first strategy, where the branch where $p \rightarrow_{j} q$ is imposed is explored first. The question remains how to select the items $p$ and $q$. We opted 
to pick those items $p$ and $q$ for which $\sum_{j \in B} \sum_{S: S \supseteq\{p, q\} \wedge p \rightarrow{ }_{j} q} y(S, j)$ is closest to 0.5 .

\subsubsection{Solving a tree node}

Before we can start solving a node of the tree, we remove all columns that do not satisfy the latest branching decision. In case of backtracking, this branching decision expires and those columns are re-entered into the model, since we experienced that they often turn out to be useful in other branches of the tree.

The LP objective value of the node can be used as an upper bound to the integral solution that could be found further down the tree. Clearly, if this value is lower than the incumbent found so far, the node can be pruned. It may, however, require a large number of iterations to prove LP optimality. Vanderbeck \& Wolsey (1996) show that the Lagrangian relaxation can also be used as an upper bound. The Lagrangian upper bound can be computed as (see e.g. Beliën (2006))

$$
\delta+\sum_{j \in B} \max _{S \subseteq G}(R C(S, j), 0)
$$

where $\delta$ is the objective value of the restricted master and $R C(S, j)$ is the reduced cost of variable $y(S, j)$. Notice that the computation of this bound requires little additional computational effort, since the pricing problem, which is solved for every bidder $j$ anyway, finds the variable with the highest reduced cost. This upper bound is referred to as the Lagrangian upper bound, since it equals the bound obtained by Lagrange relaxation (Lasdon 1970). If at any iteration in the column generation process, the Lagrangian upper bound is lower than the incumbent, we can prune the node, without any risk of missing the optimal solution.

Obviously, when we re-optimize the restricted master problem, we also start from the optimal base of the previous iteration. The first restricted master problem is solved starting from the base solution of the parent node. Furthermore, as in the root node, we delete the added columns that turned out not to be useful.

\section{Computational results}

In this section, we elaborate on how we generated the instances on which the branch-and-price algorithms were tested. We also give an overview of the computational results and compare them with results from a branch-and-cut approach performed on the assignment formulation. 


\subsection{Structure of the instances}

Unfortunately, real-life data for combinatorial auctions are not abundantly available for the public. It is therefore not uncommon in combinatorial auction literature to turn to randomly generated data (see for instance Leyton-Brown, Shoham \& Tennenholtz (2000), Sandholm (2002), and Parkes (1999)). For a thorough discussion on the empirical hardness of several data distributions commonly used for combinatorial auctions, we refer to Leyton-Brown, Nudelman \& Shoham (2005).

For our instances, each matrix bid is composed according to a bid type, randomly chosen out of the six possibilities discussed in Day (2004) (additive preference bids, single-minded bids, nested flat bids, nested k-of bids, partition bids, and add-on bids) and a bid type that has non-increasing rows and columns. In order to avoid auctions for which the exact solution of the winner determination problem is obvious, the matrix bids are constructed such that they are competitive. Furthermore, there is a parameter $H$ that bounds the highest incremental value an item brings to a set. For more details on the bid types or on how the instances were generated, we refer to Day (2004).

We performed experiments on matrix bid auctions with 5, 10, 25 or 50 items and $5,10,25,50,75$ or 100 bidders. For each combination, 10 instances were generated and solved to optimality. The highest incremental value per item $(H)$ was limited to 10 . We have no indication that the branch-and-price algorithm performs differently with other settings for $H$. All computational experiments were done on a desktop computer with a Pentium $I V 2 \mathrm{GHz}$ processor, with 512 MB RAM.

\subsection{Results}

Tables 1 and 2 give an overview of the average computation times needed to solve the matrix bid auction winner determination problem using branch-andprice with branching on an item-bidder pair (BOI) and branch-and-price with branching on a pair of successive of items (BOS) respectively. In Table 3, we give the average computation times that resulted from solving the assignment based formulation (2)-(6) with the Ilog Cplex 8.1 branch-and-cut algorithm with standard settings (B\&C), which is basically the approach followed in Day \& Raghavan (2006). Horizontally, the number of bidders $n$ varies from 5 to 100 , while the number of items $m$ auctioned ranges from 5 to 50 vertically. All computation times are expressed in seconds.

As could be expected, the computation time is determined more by the number of items in the auction, than by the number of bidders. All instances with up to 10 items are solved in less than a second by all algorithms; here the branchand-price algorithms clearly perform better. Auctions with 50 items are also solved in less than 20 minutes on average by all algorithms. The branch-and-cut algorithm seems on average the fastest way to solve these instances. Perhaps surprisingly, for the branch-and-price algorithms, the computation times for the 25 and 50 item instances do not always increase when more bidders come into 


\begin{tabular}{|r|rrrrrr|}
\hline & $n=5$ & 10 & 25 & 50 & 75 & 100 \\
\hline$m=5$ & 0.005 & 0.007 & 0.008 & 0.017 & 0.027 & 0.038 \\
10 & 0.027 & 0.038 & 0.053 & 0.088 & 0.118 & 0.169 \\
25 & 0.636 & 0.597 & 1.157 & 4.292 & 12.704 & 49.155 \\
50 & 247.224 & 60.711 & 437.951 & 557.083 & 622.591 & 802.483 \\
\hline
\end{tabular}

Table 1: Average computation times $[\mathrm{s}]$ for $n$ bidders and $m$ items using BOI

\begin{tabular}{|r|rrrrrr|}
\hline & $n=5$ & 10 & 25 & 50 & 75 & 100 \\
\hline$m=5$ & 0.005 & 0.006 & 0.006 & 0.018 & 0.027 & 0.038 \\
10 & 0.033 & 0.037 & 0.044 & 0.067 & 0.104 & 0.182 \\
25 & 0.698 & 0.767 & 1.194 & 3.814 & 16.300 & 97.122 \\
50 & 76.598 & 67.584 & 843.435 & 259.079 & 645.632 & 983.539 \\
\hline
\end{tabular}

Table 2: Average computation times [s] for $n$ bidders and $m$ items using BOS

\begin{tabular}{|r|rrrrrr|}
\hline & $n=5$ & 10 & 25 & 50 & 75 & 100 \\
\hline$m=5$ & 0.030 & 0.027 & 0.049 & 0.052 & 0.070 & 0.102 \\
10 & 0.050 & 0.069 & 0.140 & 0.278 & 0.524 & 0.748 \\
25 & 0.757 & 1.391 & 3.598 & 10.689 & 17.584 & 31.940 \\
50 & 57.676 & 28.333 & 91.230 & 215.083 & 355.785 & 811.960 \\
\hline
\end{tabular}

Table 3: Average computation times $[\mathrm{s}]$ for $n$ bidders and $m$ items using B\&C

play. This can be explained by the fact that the computation times for the individual instances tend to vary considerably.

One way to get a more accurate view on what the underlying trend is, is to consider a larger sample set. Also, it is not uncommon in literature on combinatorial auctions to study the median instead (see for instance Sandholm, Suri, Gilpin \& Levine (2005) and Hoos \& Boutilier (2000)). Tables 4 to 6 give an overview of the median computation times needed to solve the winner determination problem. The tables shows a clear trend of how the computation times rise with the number of bidders and the number of items, since the median is less affected by extreme values. It is also confirmed that the branch-and-price algorithms manage to solve the majority of the instances with many items a lot faster than reflected by the average computation times. The branch-and-cut algorithm seems to suffer less from instances with extreme computation times, since the median computation time is much closer to the average computation time. The results show that computation times for the branch-and-price algorithm with branching on an item-bidder pair rise more severely with an increasing number of items than those of the branch-and-cut algorithm. On the other hand, the branch-and-price algorithm with branching on an item-bidder pair handles an increasing number of bidders better than the branch-and-cut 


\begin{tabular}{|r|rrrrrr|}
\hline & $n=5$ & 10 & 25 & 50 & 75 & 100 \\
\hline$m=5$ & 0.000 & 0.010 & 0.010 & 0.020 & 0.030 & 0.040 \\
10 & 0.015 & 0.020 & 0.055 & 0.055 & 0.105 & 0.130 \\
25 & 0.480 & 0.460 & 0.760 & 2.445 & 9.480 & 16.825 \\
50 & 20.855 & 29.105 & 45.605 & 129.870 & 227.370 & 353.970 \\
\hline
\end{tabular}

Table 4: Median computation times [s] for $n$ bidders and $m$ items using BOI

\begin{tabular}{|r|rrrrrr|}
\hline & $n=5$ & 10 & 25 & 50 & 75 & 100 \\
\hline$m=5$ & 0.000 & 0.010 & 0.010 & 0.020 & 0.030 & 0.040 \\
10 & 0.015 & 0.020 & 0.040 & 0.055 & 0.100 & 0.130 \\
25 & 0.485 & 0.495 & 0.815 & 2.445 & 6.790 & 13.605 \\
50 & 20.855 & 29.215 & 37.970 & 129.870 & 238.785 & 514.370 \\
\hline
\end{tabular}

Table 5: Median computation times [s] for $n$ bidders and $m$ items using BOS

\begin{tabular}{|r|rrrrrr|}
\hline & $n=5$ & 10 & 25 & 50 & 75 & 100 \\
\hline$m=5$ & 0.020 & 0.025 & 0.040 & 0.050 & 0.070 & 0.105 \\
10 & 0.040 & 0.060 & 0.140 & 0.260 & 0.535 & 0.740 \\
25 & 0.530 & 1.235 & 3.245 & 10.595 & 18.120 & 28.960 \\
50 & 14.665 & 22.615 & 73.035 & 191.670 & 350.340 & 589.940 \\
\hline
\end{tabular}

Table 6: Median computation times [s] for $n$ bidders and $m$ items using B\&C

algorithm. Furthermore, apart from a couple of exceptions, the median computation times are lower with branch-and-price than with branch-and-cut.

Tables 7 and 8 give the average computation times for solving the LP-relaxation of the set packing formulation (7)-(10) and the assignment formulation (2)-(6) respectively. Recall that the former is used in both branch-and-price algorithms, while the latter is used in the branch-and-cut algorithm. The tables might be influenced by the fact that the LP-relaxation of both formulations can be solved in polynomial time. Furthermore, with two exceptions, the LP-relaxation of the set packing formulation is solved faster than the the LP-relaxation of the assignment formulation. Between brackets, the number of instances out of 10 for which the LP-relaxation resulted in an integral solution is indicated. Notice that Theorem 3 does not imply that these numbers should be at least as high for the assignment formulation than for the set packing formulation. Indeed, if there exists an integral optimal solution, the algorithms may not find it as there may be fractional solutions with the same objective value. Further, the number of instances for which an integral optimal solution was found remains more or less constant over the bidders, while it drops for instances with more items. Not surprisingly, instances with an integral LP-relaxation have low computation times. Therefore, the figures in Table 7 partially explain the fluctuations in aver- 


\begin{tabular}{|r|rrrrrr|}
\hline & $n=5$ & 10 & 25 & 50 & 75 & 100 \\
\hline$m=5$ & $0.01[8]$ & $0.01[8]$ & $0.01[7]$ & $0.02[9]$ & $0.03[10]$ & $0.04[10]$ \\
10 & $0.01[6]$ & $0.02[9]$ & $0.03[4]$ & $0.05[8]$ & $0.09[8]$ & $0.14[7]$ \\
25 & $0.31[3]$ & $0.41[7]$ & $0.80[8]$ & $2.12[8]$ & $3.29[5]$ & $4.71[4]$ \\
50 & $14.02[5]$ & $14.96[6]$ & $32.93[4]$ & $74.46[7]$ & $124.29[5]$ & $116.19[4]$ \\
\hline
\end{tabular}

Table 7: Average computation times [s] for the LP-relaxation of the set packing formulation for $n$ bidders and $m$ items

\begin{tabular}{|r|rrrrrr|}
\hline & $n=5$ & 10 & 25 & 50 & 75 & 100 \\
\hline$m=5$ & $0.01[8]$ & $0.02[9]$ & $0.02[8]$ & $0.03[8]$ & $0.04[10]$ & $0.04[9]$ \\
10 & $0.02[6]$ & $0.03[9]$ & $0.05[5]$ & $0.09[7]$ & $0.14[7]$ & $0.20[8]$ \\
25 & $0.28[5]$ & $0.51[8]$ & $1.61[7]$ & $4.47[9]$ & $6.03[5]$ & $8.35[5]$ \\
50 & $9.40[5]$ & $20.01[6]$ & $43.93[3]$ & $159.46[6]$ & $313.76[4]$ & $461.91[4]$ \\
\hline
\end{tabular}

Table 8: Average computation times $[\mathrm{s}]$ for the LP-relaxation of the assignment formulation for $n$ bidders and $m$ items

age computation times for the instances with 25 or 50 items (see Tables 1 and 2).

Finally, Table 9 gives an overview of the performance details of the three algorithms. Column $A$ gives the average number of nodes in the branching tree that were explored. Column $B$ represents the average number of pricing rounds, and column $C$ gives the average number of variables that were generated (these columns are not applicable for the branch-and-cut algorithm). On the rows, we find the instances, where the first number indicates the number of items and the second gives the number of bidders. There seems to be no systematic difference between the branch-and-price algorithms for any of the three parameters described in this table. The branch-and-cut algorithm solves very little nodes in its branching tree, compared to the branch-and-price algorithms. In many cases, the branch-and-cut algorithm prefers generating valid inequalities in the root node to branching.

\section{Conclusion}

In this paper, we studied the winner determination problem for the matrix bid auction. We first looked at a special case of the matrix bid auction, namely where all bidders have an identical ranking of the items. For this auction, we found that that there exists no polynomial-time approximation scheme for the winner determination problem, unless $P=N P$. However, there exists a polynomial time algorithm in the case the number of bidders is fixed. Then, we compared two mathematical formulations for the winner determination problem of the general matrix bid auction. One assignment is based on the assignment problem, while the other is based on the set packing problem. We found 


\begin{tabular}{|c|c|c|c|c|c|c|c|}
\hline \multirow[b]{2}{*}{ Inst. } & \multicolumn{3}{|c|}{ BOI } & \multicolumn{3}{|c|}{ BOS } & \multirow{2}{*}{$\begin{array}{c}\text { B\&C } \\
A\end{array}$} \\
\hline & $\mathrm{A}$ & B & $\mathrm{C}$ & $\mathrm{A}$ & B & $\mathrm{C}$ & \\
\hline $5-5$ & 2.2 & 3.9 & 33.3 & 2.4 & 4.7 & 34.4 & 1.0 \\
\hline $5-10$ & 1.3 & 3.7 & 68.4 & 1.4 & 3.4 & 68.3 & 1.0 \\
\hline $5-25$ & 2.5 & 4.5 & 142.2 & 1.6 & 3.2 & 141.6 & 1.0 \\
\hline $5-50$ & 1.3 & 3.2 & 266.9 & 1.2 & 3.1 & 266.9 & 1.0 \\
\hline $5-75$ & 1.0 & 2.4 & 435.9 & 1.0 & 2.4 & 435.9 & 1.0 \\
\hline $5-100$ & 1.0 & 2.0 & 565.0 & 1.0 & 2.0 & 565.0 & 1.0 \\
\hline $10-5$ & 7.6 & 18.3 & 124.2 & 9.4 & 27.5 & 93.7 & 1.2 \\
\hline $10-10$ & 7.6 & 16.5 & 201.8 & 5.4 & 16.8 & 193.8 & 1.5 \\
\hline $10-25$ & 4.9 & 10.2 & 363.8 & 2.8 & 8.4 & 352.0 & 1.0 \\
\hline $10-50$ & 3.7 & 8.0 & 788.9 & 1.8 & 5.4 & 782.2 & 1.0 \\
\hline $10-75$ & 2.3 & 6.9 & $1,117.7$ & 1.6 & 6.0 & $1,113.8$ & 1.0 \\
\hline $10-100$ & 2.3 & 7.0 & $1,459.5$ & 2.6 & 8.4 & $1,455.4$ & 1.0 \\
\hline $25-5$ & 7.7 & 72.3 & $1,017.7$ & 6.4 & 89.4 & 723.1 & 1.2 \\
\hline $25-10$ & 2.0 & 37.8 & 990.7 & 6.8 & 51.7 & 864.5 & 1.5 \\
\hline $25-25$ & 4.4 & 31.2 & $1,793.3$ & 3.8 & 33.2 & $1,752.1$ & 1.0 \\
\hline $25-50$ & 8.6 & 59.7 & $3,703.2$ & 5.2 & 55.1 & $3,602.4$ & 1.0 \\
\hline $25-75$ & 30.0 & 123.5 & $5,402.7$ & 32.8 & 143.1 & $5,412.7$ & 1.0 \\
\hline $25-100$ & 96.8 & 349.3 & $7,564.0$ & 163.0 & 635.3 & $7,895.2$ & 1.3 \\
\hline $50-5$ & 21.4 & $3,095.8$ & $4,745.9$ & 37.9 & $1,279.2$ & $2,872.6$ & 11.8 \\
\hline $50-10$ & 12.2 & 592.6 & $3,963.3$ & 27.9 & 611.7 & $4,112.8$ & 1.0 \\
\hline $50-25$ & 315.1 & $1,494.0$ & $11,752.4$ & $1,029.6$ & $2,806.7$ & $10,141.0$ & 1.2 \\
\hline $50-50$ & 361.6 & 938.5 & $16,278.1$ & 67.6 & 468.4 & $14,359.3$ & 1.0 \\
\hline $50-75$ & 102.5 & 828.5 & $20,773.4$ & 106.7 & 852.0 & $20,776.0$ & 1.0 \\
\hline $50-100$ & 96.0 & 839.5 & $30,538.0$ & 100.4 & 995.4 & $31,120.3$ & 5.7 \\
\hline
\end{tabular}

Table 9: Performance details for the three algorithms (BOI, BOS, B\&C) 
that both formulations are equally strong. Moreover, an integral solution for one formulation can always be translated to an integral solution for the other formulation. We used the set packing formulation as a basis for a column generation approach where the pricing problem can be solved as a shortest path problem. This means that we are able to solve the LP-relaxation of the set packing formulation in polynomial time. We then extended this approach to two branch-and-price algorithms. In one algorithm, we branch on the items, while in the other, branching is done on the succession of the items. The pricing problem for these branch-and-price algorithms remains solvable as a shortest path problem throughout the search tree. These algorithms are tested on randomly generated instances with up to 50 items and 100 bidders, which they solved within 20 minutes (on average). Finally, the branch-and-price algorithms withstood the comparison with a branch-and-cut algorithm, based on Day \& Raghavan (2006). The algorithms perform better on instances with up to 10 items, but are outperformed by the branch-and-cut algorithm on some of the larger instances. The increase in computation time, however, seems favorable for the branch-and-price algorithms, which indicates that they form at least a viable approach to solve instances of the matrix bid auction winner determination problem.

\section{References}

Barnhart, C., Johnson, E. L., Nemhauser, G. L., Savelsbergh, M. W. P. \& Vance, P. H. (1998). Branch-and-price: column generation for solving huge integer programs, Operations Research 46: 316-329.

Beliën, J. (2006). Exact and heuristic methodologies for scheduling in hospitals: problems, formulations and algorithms, $\mathrm{PhD}$ thesis, K.U.Leuven.

Bleischwitz, Y. \& Kliewer, G. (2005). Accelerating Vickrey payment computation in combinatorial auctions for an airline alliance., in WEA '05: Proceedings of the 4 th international workshop on experimental and efficient algorithms, Santorini Island, Greece, Springer, pp. 228-239.

Dantzig, G. B. \& Wolfe, P. (1960). Decomposition principle for linear programs, Operations Research 8: 101-111.

Day, R. W. (2004). Expressing preferences with price-vector agents in combinatorial auctions, $\mathrm{PhD}$ thesis, University of Maryland.

Day, R. W. \& Raghavan, S. (2006). Matrix bidding in combinatorial auctions. Manuscript.

de Vries, S. \& Vohra, R. V. (2003). Combinatorial auctions: a survey, INFORMS Journal on Computing (3): 284-309.

Goossens, D., Maas, A. J. T., Spieksma, F. C. R. \& van de Klundert, J. J. (2007). Exact algorithms for procurement problems under a total quantity discount structure, European Journal of Operational Research 178(2): 603626 . 
Goossens, D. \& Spieksma, F. C. R. (2006). The matrix bid auction: microeconomic properties and expressiveness, FETEW Research Report KBI0619, K.U.Leuven.

Hoos, H. \& Boutilier, C. (2000). Solving combinatorial auctions using stochastic local search, in AAAI/IAAI '00: Proceedings of the 17th national conference on artificial intelligence and 12th conference on innovative applications of artificial intelligence, Austin, USA, AAAI Press/The MIT Press, pp. 22-29.

Kann, V. (1991). Maximum bounded 3-dimensional matching is max snpcomplete, Information Processing Letters 37(1): 27-35.

Lasdon, L. (1970). Optimization theory for large systems, MacMillan.

Leyton-Brown, K., Nudelman, E. \& Shoham, Y. (2005). Empirical hardness models for combinatorial auctions, in P. Cramton, R. Steinberg \& Y. Shoham (eds), Combinatorial auctions, MIT Press, pp. 479-504.

Leyton-Brown, K. \& Shoham, Y. (2005). A test suite for combinatorial auctions, in P. Cramton, R. Steinberg \& Y. Shoham (eds), Combinatorial auctions, MIT Press, pp. 451-478.

Leyton-Brown, K., Shoham, Y. \& Tennenholtz, M. (2000). An algorithm for multi-unit combinatorial auctions, in AAAI/IAAI '00: Proceedings of the 17th national conference on artificial intelligence and 12th conference on innovative applications of artificial intelligence, Austin, USA, AAAI Press / The MIT Press, pp. 56-61.

Moonen, L. (2005). Algorithms for some graph-theoretical optimization problems, $\mathrm{PhD}$ thesis, K.U.Leuven.

Nisan, N. (2000). Bidding and allocation in combinatorial auctions, in EC '00: Proceedings of the 2nd ACM conference on electronic commerce, Minneapolis, USA, ACM Press, pp. 1-12.

Parkes, D. C. (1999). iBundle: an efficient ascending price bundle auction, in EC '99: Proceedings of the ACM conference on electronic commerce, Denver, USA, ACM Press, pp. 148-157.

Petrank, E. (1994). The hardness of approximation: gap location, Computational Complexity 4(2): 133-157.

Rothkopf, M., Pekeç, A. \& Harstad, R. M. (1998). Computationally manageable combinational auctions, Management Science 44(8): 1131-1147.

Ryan, D. \& Foster, B. (1981). An integer programming approach to scheduling, in A. Wren (ed.), Computer scheduling of public transport: urban passenger vehicle and crew scheduling, North-Holland, pp. 269-280.

Sandholm, T. (2002). Algorithm for optimal winner determination in combinatorial auctions, Artificial Intelligence 135(1-2): 1-54. 
Sandholm, T., Suri, S., Gilpin, A. \& Levine, D. (2005). CABOB: a fast optimal algorithm for combinatorial auctions, Management Science 51: 374390.

Tennenholtz, M. (2002). Tractable combinatorial auctions and b-matching, Artificial Intelligence 140(1/2): 231-243.

Vanderbeck, F. \& Wolsey, L. (1996). An exact algorithm for ip column generation, Operations Research Letters 19: 151-159. 